\title{
Estilos de liderazgo y engagement laboral en analistas de crédito del sector financiero en Arequipa
}

\author{
Leadership styles and labor engagement in credit analysts of the financial \\ sector in Arequipa
}

\author{
José Arias-Gonzáles 1,a \\ https://orcid.org/0000-0002-3250-5287
}

\section{Citar como}

Arias-Gonzáles, José (2021). Desafíos, 12(1); 09-15. https://doi.org/10.37711/desafios.2021.12.1.256

\section{RESUMEN}

Objetivo. El objetivo fue determinar cómo se relacionan los "estilos de liderazgo" y el "engagement laboral" de los analistas de crédito en las empresas del sector financiero en Arequipa, 2020. Métodos. Se realizó un estudio básico, con un alcance correlacional y un diseño no experimental-transversal. La población estuvo conformada por 268 analistas de crédito y se utilizó el muestreo no probabilístico para establecer la muestra que quedó fijada en 253 analistas de crédito. La técnica es la encuesta y los instrumentos son el cuestionario CELID-A y la escala UWES. Resultados. Los resultados demuestran que el estilo de liderazgo transaccional se relaciona significativamente con un nivel de "engagement laboral" alto $(p$-valor $=0,00)$. El estilo de liderazgo Laissez faire se relaciona significativamente con un nivel de "engagement laboral" bajo $(0,01)$ y el estilo de liderazgo transformacional no se relaciona con el "engagement laboral" $(0,13)$. Conclusión. Existe relación entre los "estilos de liderazgo" y el "engagement laboral" de los analistas de crédito en las empresas del sector financiero.

Palabras clave: liderazgo; crédito; compromiso.

\begin{abstract}
Objective. Determine the relationship between "leadership styles" and "work engagement" of credit analysts in companies in the financial sector in Arequipa, 2020.. Methods. A basic study was carried out, with a correlational scope and a non-experimental-cross-sectional design. The population consisted of 268 credit analysts and the non-probabilistic sampling was used to establish the sample that was set at 253 credit analysts. The technique used was the survey and the instruments were the CELID-A questionnaire and the UWES scale. Results. The results show that the transactional leadership style is significantly related to a high level of "work engagement" $(p$-value $=0.00)$. Laissez faire leadership style is significantly related to a low level of "work engagement" (0.01) and transformational leadership style is not related to "work engagement" (0.13). Conclution. There is a relationship between "leadership styles" and "work engagement" of credit analysts in companies in the financial sector.

Keywords: leadership; credit; commitment.
\end{abstract}




\section{INTRODUCCIÓN}

Las empresas se definen como un organismo social y de continuidad compuesto por partes interrelacionadas entre sí. El contexto en el que se organiza la empresa depende de cada una de sus partes, funcionando de forma sistemática, coherente y con continuidad. Las empresas, entendidas desde esta perspectiva, proporcionan un conjunto de elementos en interacción dinámica que empieza en una cabeza (el líder) hasta los pies (los colaboradores); por supuesto, no debe verse como un sistema de mejor-peor o mayor-menor, sino como un conjunto de elementos funcionando para una misma causa. En consecuencia, las acciones de los involucrados generan el desarrollo de cualquier empresa (Ganga y Navarrete, 2013).

Para el eficiente funcionamiento de una empresa existenequipos detrabajo,quienes sonconformados por el talento humano. Estos, normalmente, se organizan de forma jerárquica y tienen la principal característica de cooperar, integrar, sonetizar, compartir capacidades, aptitudes, actitudes, experiencias e información para lograr el propósito de la organización; asimismo, en todo equipo existe un líder o una persona que tiene funciones de liderazgo para llevar el equipo al cumplimiento las metas organizacionales (Bourgault y Daoudi, 2014). El líder es la persona que tiene la capacidad de establecer visones y metas, organizar acciones, crear y asignar normas, y orientar las actividades de su equipo; esto implica que el líder debe poseer capacidades que le permiten tener una imagen privilegiada, logrando que el grupo lo acepte, lo obedezca y acepte las disposiciones de este (Díaz et al, 2018).

El rol del liderazgo está relacionado con la promoción del crecimiento organizacional e individual de la empresa, y permite el desarrollo de habilidades y capacidades en todos los integrantes del equipo; siendo necesario para ello que el líder cuente con habilidades sociales y cognitivas que le permitan realizar aportes, gestionar y desarrollar equipos de trabajos eficientes. A las organizaciones les interesa crear colaboradores con liderazgo que incrementen sus potencialidades para el análisis del entorno y fortalezcan la transformación en la organización para así dar respuesta a las distintas exigencias y demandas del entorno (Aguirre et al, 2016).

Justamente, para lograr el cumplimiento de las metas, el crecimiento continuo de la organización, el desarrollo de las capacidad y habilidades, es menesterquelostrabajadoresesténcomprometidos con dichas metas; a este compromiso es al que que se le conoce como engagement. Leiter y Maslach definen el engagement como un estado energético relacionado con las actividades que desempeña cada quien para fortalecer su desempeño profesional. Los trabajadores con engagement son personas que toman iniciativas individuales en las labores que realizan, desarrollando su propio feedback sobre su desempeño; por lo tanto, están en la constante búsqueda de nuevos desafíos y se sienten comprometidos en tener un óptimo desempeño (Sanclemente et al, 2017).

Aunque resulta complejo definir específicamente el engagement, López y Chiclana (2017) lo definen como el aprovechamiento de los integrantes de la organización con las propias funciones de sus labores; por medio del engagement, las personas emplean y se expresan a sí mismas de forma física, cognitiva, emocional y de forma mental durante el desempeño de sus funciones.

Según Villaba y Fierro (2017), el engagement está asociado al altruismo organizacional, sentido de colaboración y la unión óptima del trabajador con su labor. Este se describe además como una unión psicológica hacia el trabajo y dicha unión de las personas da respuesta a la relación de los recursos laborales y personales, la respuesta de los mismos al esfuerzo, las capacidades y habilidades percibidas por las propias personas para el cumplimiento de sus tareas en el ámbito laboral, además de la propia percepción de la eficiencia profesional y el contagio emocional fuera del trabajo. Además, todas estas características funcionan como un activador del trabajo.

Con respecto a lo manifestado previamente, estudios realizados por Pérez y Valderrama (2018) sobre los "estilos de liderazgo", felicidad y relación con el engagement en una organización del Gobierno Regional de Arequipa, la Autoridad Autónoma de Majes, Siguas, permitieron establecer la existencia de una relación positiva ente los "estilos de liderazgo" y el engagement $(R=0,568 ; p$ $<0,05)$. Otro estudio realizado por Zumaeta (2018) sobre la relación entre los "estilos de liderazgo" y engagement en trabajadores de una empresa textil en Lima, establecieron que el estilo transformacional se relaciona de forma positiva y significativa con el "engagement laboral" con un coeficiente de correlación rho de Spearman de 0,669, seguido por el estilo transaccional con un valor obtenido de .661.

Un estudio similar realizado por Bratzo (2018) sobre la relación entre los "estilos de liderazgo" y el compromiso organizacional permitió conocer que, los "estilos de liderazgo" presentaron una correlación significativa con el compromiso organizacional, siendo el estilo transaccional el que 
presenta mayor asociación con el compromiso organizacional. Por último, un artículo presentado por Martínez et al. (2016) sobre el liderazgo como promotor del engagement en el trabajo, determinó que existe una relación significativa y positiva entre el liderazgo directivo y el engagment en el trabajo.

El liderazgo es una herramienta intrínseca en un individuo que puede producir cambios significativos en los colaboradores y la empresa, ergo, existen distintos "estilos de liderazgo" que producen cambios negativos como la rotación del personal o la disminución del nivel de productividad de los colaboradores y, asimismo, estilos de liderazgo que pueden producir cambios positivos como la eficiencia del trabajo en equipo, un clima laboral positivo o el compromiso laboral de los trabajadores.

El presente estudio se realiza porque es relevante que los trabajadores se sientan comprometidos con los propósitos y fines de la empresa, dado que esto es uno de los factores principales del crecimiento de la organización; sin embargo, todo depende de la línea jerárquica, teniendo en cuenta que existen gerentes o jefes que deben tener la capacidad para liderar e influir para que estos trabajadores se comprometan con la empresa. Estudiar la asociación entre estos estilos de liderazgo a través del engagement laboral, permite alinear el estilo que se requiere para conectar a estos empleados y buscar un objetivo común. Es por tal razón que el objetivo general de la presente investigación fue determinar cómo se relacionan los "estilos de liderazgo" y el "engagement laboral" de los analistas de crédito en las empresas del sector financiero en Arequipa, 2020

\section{"estilos de liderazgo"}

Para Aguirre et al. (2016), "El liderazgo es la capacidad de influir en los demás para el alcance de los objetivos" (p. 188). Por otro lado, para Vásquez et al. (2014), "el liderazgo es una habilidad que necesita ser aprendida y es una cualidad que debe ser desarrollada" (p. 83). Ser líder no es algo innato, por el contrario, requiere del desarrollo de ciertas habilidades y capacidades que deben ser aprendidas por la persona; es decir, la persona debe aprender a tener y utilizar el liderazgo.

Los "estilos de liderazgo" pueden ser reconocidos por sus rasgos, filosofía y el grupo de habilidades propias de la gestión. Las características de los "estilos de liderazgo" detallan la relevancia del líder para el logro del rendimiento; más aún, la filosofía define la manera en que influye el líder en las personas y el rol de este. Las habilidades agrupan las capacidades de la gestión que son propias de un liderazgo determinado (Escandon y Hurtado,
2016). El estilo de liderazgo forma parte de los modelos de conducta que es visto por las demás personas, además del punto de vista del propio del líder en función a su posición y actuación, y esto es lo que hace que los miembros del equipo actúen y ejecuten las acciones que se les solicita (Rodríguez et al, 2017).

Kurt Lewin (1939) y Bass Avolio (1994) plantean los siguientes "estilos de liderazgo":

a) Transformacional: en este estilo, el líder genera propósitos y metas como retos, los cuales se pretenden lograr como institución o como equipo; para ello, el líder les da poder a sus subordinados y construye un entorno laboral fundamentado en los valores organizacionales y reconoce las habilidades de cada uno de los integrantes del equipo. Los líderes transformacionales alcanzan una influencia idealizada basada en el carisma, produce confianza y admiración entre sus seguidores, es respetado e imitado por los seguidores, genera un clima cooperativo, logra la motivación intelectual de sus subordinados, estimula y guía a sus subordinados.

b) Transaccional: este líder genera un acuerdo con sus subordinados en función a los objetivos y recompensas; unido a esto se encuentra la supervisión y el control que le realiza el líder a los seguidores con la finalidad de medir las metas y objetivos acordados. El líder transaccional se caracteriza por generar recompensas contingentes, donde el líder es el que determina lo que se va hacer, como se va hacer y las recompensas que la acción debe generar. El líder supervisa el progreso de sus subordinados y, en caso de que exista alguna desviación, utiliza la reorientación para encaminarlos nuevamente al proceso

c) Laissez faire: el líder no realiza las funciones que le corresponden, no muestra responsabilidad por el grupo y los deja a cargo de su propia iniciativa (Sánchez y Barraza, 2015). En este estilo de liderazgo, el líder intenta evitar las decisiones y acciones que lo responsabilizan de los resultados. Es un liderazgo donde no existe autoridad, generando con ello una ausencia física y psicológica de la gerencia en momentos difíciles e impidiendo el control y orientación de los subordinados (Bernasconi y Rodríguez, 2018).

\section{Engagement laboral}

El engagement es un estado psicológico asociado con la antítesis positiva del burnout, desde donde se describe como un estado mental positivo, de realización y asociado con la labor, el cual se encuentra determinado por los rasgos de vigor, dedicación y absorción (Marcantonio, 
2017). El "engagement laboral" es una necesidad enfocada en el fortalecimiento del bienestar de los miembros de la organización, ya que se orienta a la interpretación y compresión de las fortalezas de las personas, su relación con el entono y su desempeño como trabajadores (Pérez y Pedraza, 2018).

El engagement se encuentra determinado por tres factores esenciales, los cuales son:

- Vigor: elevados índices de energía y resistencia mental por el deseo de realizar esfuerzo en la labor que se ejecuta, aun cuando existen obstáculos.

- Dedicación: niveles altos de relación laboral, aunado a un evidente sentimiento significativo, entusiasmo, honra y reto por la labor.

- Absorción: la persona se encuentra en un alto estado de concentración con la labor que realiza, lo que hace que sienta que el tiempo pasa muy rápido y tenga problemas al momento de dejar lo que está haciendo producto de la sensación de satisfacción y disfrute en lo que siente (Marcantonio, 2017).

\section{MÉTODOS}

Tipo de estudio. La metodología de la investigación se basó en una investigación básica, la cual se caracteriza por incrementar el conocimiento y principios teóricos fundamentales de las variables de estudio (Hernández-Sampieri y Mendoza, 2018). El alcance de la investigación fue el correlacional, el cual tiene la característica de asociar o relacionar las variables de estudio y no existe causa-efecto entre las variables, pero es una condición que se pueda analizar por el investigador (Arias, 2020). El diseño de la investigación fue no Experimentaltransversal (Hernández-Sampieri y Mendoza, 2018); este tipo de investigación se da cuando no se van a manipular variables y se va a realizar en un único periodo.

Población y muestra. La población estuvo constituida por 268 analistas de crédito de siete empresas del sector financiero en Arequipa, y mediante el muestreo no probabilístico-intencional, la muestra quedó conformada por 253 analistas de crédito de las siete empresas del sector financiero en Arequipa.

Instrumentos de recolección de datos. La técnica que se utilizó para ambas variables fue la encuesta y el instrumento para la variable "estilos de liderazgo" fue el cuestionario CELID-A, con un valor de confiabilidad estadística alfa de Cronbach de 0,75. Para la variable "engagement laboral" se utilizó la escala Ultrecht Work Engagement Scale
(UWES), elaborada por Sonia Palma, con un valor de confiabilidad alfa de Cronbach de 0,93.

Procedimientos de la recolección de datos. La recolección de datos se realizó de forma virtual; para ello, se utilizó una herramienta digital de Google Ilamada Google Forms y se siguió el siguiente procedimiento según Arias (2020): Elegir la herramienta digital acorde al tipo de instrumento; tener la base de datos, correos, celular, u otro medio que permita el contacto con los participantes de estudio; transferir las preguntas o enunciados y las opciones de respuesta de forma idéntica del cuestionario físico al instrumento virtual.

Análisis de datos. Los resultados fueron sistematizados en Excel 2016, desde donde se generaron las tablas de distribución y figuras para luego exportarlas a Word 2016. Para elegir la prueba de hipótesis se estableció que las variables son categóricas y tienen una escala de medición ordinal; además, se aplicó una prueba de normalidad bajo los parámetros de kolmorogov-Smirnov, quedando como resultado un nivel de significancia de 0,00; lo que indica una distribución no normal de la población. Teniendo en cuenta estos parámetros, se decide usar la prueba estadística chi cuadrado, la cual fue procesada en el sistema IBM SPSS stadistic V25.0.

La hipótesis planteada (Hi) fue la siguiente: los "estilos de liderazgo" se relacionan significativamente con el "engagement laboral" de los analistas de crédito en las empresas del sector financiero en Arequipa, 2020

Aspectos éticos. El estudio se presenta con un diseño no experimental; por tal razón, el proceso de recolección de datos no impactó de forma negativa en los participantes del estudio; además, los resultados son anónimos, por lo que los participantes no se ven vulnerados en su identidad. Asimismo, se dispuso un consentimiento informado en el que detalla los fines del estudio.

\section{RESULTADOS}

Para presentar los resultados, se toman los tres "estilos de liderazgo" segúnel modelo de Bassy Avolio (1994): estilo transformacional, estilo transaccional y estilo Laissez faire. Se presentan resultados por cada variable de estudio y se van a asociar los tres "estilos de liderazgo" con el "engagement laboral" para identificar el estilo de liderazgo que se relaciona con un alto nivel de "engagement laboral" y el estilo de liderazgo que se relaciona con un bajo nivel de "engagement laboral". 
Tabla 1

"Estilos de liderazgo" en las empresas del sector financiero en Arequipa

\begin{tabular}{lcc}
\hline Estilos & $\mathrm{fi}$ & $\%$ \\
\hline Transformacional & 29 & $11,5 \%$ \\
Transaccional & 152 & $60,1 \%$ \\
Laissez faire & 72 & $28,5 \%$
\end{tabular}

Nota. Sistematización de los resultados de las encuestas en el programa Excel 2016.

En la tabla 1, se mostró que el estilo de liderazgo que tiene mayor presencia en las empresas del sector financiero en Arequipa es el transaccional, seguido por el Laissez faire. Los analistas de crédito transcurren más de la mitad de su jornada laboral fuera de la oficina; es decir, en el campo, ofreciendo créditos y visitando a sus potenciales clientes. Esta actividad se alinea con las funciones del líder transaccional que se caracteriza por plantear metas semanales y mensuales, y supervisar el progreso de los resultados obtenidos.

En la tabla 2, se evidenció que el nivel de "engagement laboral" de más de la mitad de los analistas de crédito es alto; sin embargo, existe una tercera parte que tienen un nivel bajo de "engagement laboral". El hecho de que los analistas de crédito realicen su jornada laboral fuera de la oficina implica que algunos no se sientan comprometidos con la empresa. Así lo demostró también un estudio realizado por Huayta y Luque (2019), en el que los analistas de crédito afirmaron no sentir pertenencia, lealtad ni continuidad en la empresa que trabajan, debido a que su trabajo se realiza en el campo y la razón principal de hacerlo es solamente para obtener mayores beneficios económicos.

En la tabla 3, se estableció que el estilo de liderazgo transformacional se asocia, en mayor
Tabla 2

Nivel de "engagement laboral" de los analistas de crédito en las empresas del sector financiero en Arequipa

\begin{tabular}{ccc}
\hline Nivel & $\mathrm{fi}$ & $\%$ \\
\hline Bajo & 78 & $30,8 \%$ \\
Regular & 29 & $11,5 \%$ \\
Alto & 146 & $57,7 \%$ \\
\hline
\end{tabular}

Nota. Sistematización de los resultados de las encuestas en el programa Excel 2016.

medida, con un nivel de "engagement laboral" regular; sin embargo, no existió una relación significativa debido a que el valor obtenido en la prueba de chi cuadrado fue 0,13. El estilo de liderazgo transaccional se asoció con un nivel de "engagement laboral" alto y el valor obtenido en la prueba chi cuadrado de 0,00, por lo que se demostró la existencia de una relación significativa. Por último, el estilo de liderazgo Laissez faire estuvo asociado, en mayor medida, a un nivel bajo de "engagement laboral" y el valor obtenido en la prueba chi cuadrado de 0,01, se demostró la existencia de una relación significativa.

En la tabla 4, los resultados obtenidos en la prueba de hipótesis estadística chi cuadrado para las variables "estilos de liderazgo" y "engagement laboral", obtuvieron un valor de significancia de 0,003 . Con este resultado se tomó la decisión de rechazar la hipótesis nula: los "estilos de liderazgo" no se relacionan significativamente con el "engagement laboral" de los analistas de crédito en las empresas del sector financiero en Arequipa, 2020. Asimismo, se aceptó la hipótesis de investigación: los "estilos de liderazgo" se relacionan significativamente con el "engagement laboral" de los analistas de crédito en las empresas del sector financiero en Arequipa, 2020.

\section{Tabla 3}

Resultados cruzados entre los "estilos de liderazgo" y el nivel de "engagement laboral" en las empresas del sector financiero en Arequipa

\begin{tabular}{|c|c|c|c|c|c|c|}
\hline & & & \multicolumn{3}{|c|}{ "Estilos de liderazgo" } & \multirow[b]{2}{*}{ Total } \\
\hline & & & $\begin{array}{c}\text { Estilo } \\
\text { transformacional }\end{array}$ & $\begin{array}{c}\text { Estilo } \\
\text { transaccional }\end{array}$ & $\begin{array}{l}\text { Estilo Laissez } \\
\text { faire }\end{array}$ & \\
\hline \multirow{6}{*}{$\begin{array}{l}\text { "Engagement } \\
\text { laboral" }\end{array}$} & \multirow{2}{*}{ Bajo } & Recuento & 10 & 7 & 61 & 78 \\
\hline & & $\%$ del total & $4,0 \%$ & $2,8 \%$ & $24,1 \%$ & $30,8 \%$ \\
\hline & \multirow{2}{*}{ Regular } & Recuento & 15 & 9 & 5 & 29 \\
\hline & & $\%$ del total & $5,9 \%$ & $3,6 \%$ & $2,0 \%$ & $11,5 \%$ \\
\hline & \multirow{2}{*}{ Alto } & Recuento & 4 & 136 & 6 & 146 \\
\hline & & $\%$ del total & $1,6 \%$ & $53,8 \%$ & $2,4 \%$ & $57,7 \%$ \\
\hline \multirow{2}{*}{\multicolumn{2}{|c|}{ Total }} & Recuento & 29 & 152 & 72 & 253 \\
\hline & & $\%$ del total & $11,5 \%$ & $60,1 \%$ & $28,5 \%$ & $100,0 \%$ \\
\hline
\end{tabular}

Nota. Sistematizado de los resultados consolidados en el sistema IBM SPSS stadistic V25.0. 
Tabla 4

Prueba de hipótesis estadística de chi cuadrad

\begin{tabular}{lccc}
\hline & Valor & df & $\begin{array}{c}\text { Significación } \\
\text { asintótica } \\
\text { (bilateral) }\end{array}$ \\
\hline Chi-cuadrado de Pearson & $214,913 a$ & 4 & 0,003 \\
Razón de verosimilitud & 211,870 & 4 & 0,000 \\
Asociación lineal por lineal & 47,946 & 1 & 0,000 \\
N de casos válidos & 253 & & \\
$\begin{array}{l}\text { a. 1 casillas (11,1\%) han esperado un recuento menor que 5. El } \\
\text { recuento minimo esperado es 3.32. }\end{array}$
\end{tabular}

Nota. Sistematizado de los resultados consolidados en el sistema IBM SPSS stadistic V25.0.

\section{DISCUSIÓN}

Habiendo considerado la forma de trabajo de los analistas de crédito, quienes tienen como mayor incentivo de trabajo, el económico, es consecuente que tome relevancia el estilo de liderazgo transaccional en las empresas del sector financiero, debido a que este estilo se caracteriza por generar recompensas contingentes y es el que determina lo que se va hacer, como se va hacer y las recompensas que la acción debe generar. (Bernasconi y Rodríguez, 2018). Por otra parte, el nivel alto de "engagement laboral" presentado en este estudio es consecuente con el vigor y dedicación de los analistas de crédito, debido a que ellos deben cumplir con las metas planteadas por el supervisor, sin tomar en cuenta, en muchas ocasiones, el tiempo o la jornada laboral.

Los resultados demostraron que en las empresas del sector financiero en Arequipa, el 60,1\% de los analistas de crédito tienen un estilo de liderazgo transaccional, mientras que el $57,7 \%$ tienen un nivel alto de engagement laboral; asimismo, existe una relación significativa entreel liderazgo transaccional, el "engagement laboral" y el liderazgo Laissez faire con el "engagement laboral". El estilo de liderazgo transformacional no se relaciona significativamente con el "engagement laboral".

Estos resultados son similares a los obtenidos por Bratzo (2018), quién concluyó que los "estilos de liderazgo" presentan una correlación significativa con el compromiso organizacional, siendo el estilo transaccional el que presenta mayor incidencia en el compromiso organizacional. Así también, el estudio realizado por Pérez y Valderrama (2018) concluyó con la existencia de una relación positiva entre los "estilos de liderazgo" y el engagement $r=$ 0,568; $p<0,05$. Por su parte, Martínez et al. (2016) determinaron que existe una relación significativa y positiva entre el liderazgo directivo y el engagment en el trabajo. Resultados contrarios fueron obtenidos por Zumaeta (2018), quien estableció que el estilo transformacional se relaciona de forma positiva y significativa con el "engagement laboral" con un valor de 669 .

La obtención de los datos, como se expuso en la metodología del estudio, se realizó de forma virtual; esto influyó en el tiempo para recabar los resultados debido a que el tiempo de respuesta de los encuestados fue de 20 días (más de lo previsto). Algunos enviaban los resultados en los primeros días, otros lo hicieron después de algunas semanas; asimismo, hubieron dos analistas quienes, en un inicio, fueron parte del estudio, empero, al enfermar con la covid-19, no pudieron hacerlo por lo que la muestra inicial de 255 analistas de crédito, finalmente quedó conformada por 253. El estudio mide los "estilos de liderazgo", los cuales han sido planteados y abordados por diferentes autores; sin embargo, se debe tener en cuenta que hoy en día, por el trabajo remoto, existe un liderazgo virtual, el cual, debe tener nuevas competencias y formas de liderazgo. En ese sentido, es importante que se investigue la forma en la que se debe liderar a los analistas de crédito desde un ambiente remoto, además de plantear estrategias que puedan elevar los niveles de compromiso de los analistas de crédito ahora que el contacto físico con su entidad es casi nulo.

Como conclusión, en el estudio se evidencia que los analistas de crédito presentan mayor engagement laboral cuando el líder recompensa las acciones y las metas cumplidas por los analistas de crédito, mientras que, cuando el líder no toma decisiones ni acciones para cumplir con los objetivos en la otorgación de créditos, los analistas de crédito presentan menor nivel de "engagement laboral". Ahora bien, si se ha presentado el estilo de liderazgo que construye valores, confianza o un clima organizacional positivo, este estilo transformacional no genera ningún estímulo al analista de crédito, lo que se debe al tipo de trabajo de campo que realiza. Con respecto al "engagement laboral", los analistas de crédito han demostrado tener energía y resistencia mental durante su actividad laboral, además de entusiasmo, lo que se ve reflejado en su vigor y dedicación; sin embargo, esto se debe a los incentivos económicos por el cumplimiento de metas y el otorgamiento de más créditos a sus clientes.

\section{REFERÉNCIAS}

Aguirre, G., Serrano, B. y Sotomayor, G. (2016). El liderazgo de los gerentes. Universidad y Sociedad, 8(3), 187-195.

Arias, J. L. (2020). Métodos de investigación online, herramientas digitales para recolectar datos. Arequipa. 
Arias, J. L. (2020). Proyecto de tesis, guia para la elaboración. Arequipa

Bass, B. y Avolio, B. (1994). Improving organizational effectiveness through transformational leadership. Sage Publications.

Bernasconi, A. y Rodríguez, E. (2018). Análisis exploratorio de las percepciones sobre los "estilos de liderazgo", el clima académico y la caliadad de la formación de pregrado. Revista Formación Universitaria, 11(3). https://scielo.conicyt.cl/scielo.php?pi$\mathrm{d}=$ S0718-50062018000300029\&script=sci_arttext\&tlng $=e$

Bratzo, M. (2018). Relación entre los "estilos de liderazgo" y el compromiso organizacional [Tesis de pregrado, Pontificia Universidad Catolica del Perú]. Repositorio Digital de Tesis y Trabajos de Investigación PUCP. http://hdl.handle.net/20.500.12404/13624

Bourgault, M. y Daoudi, J. (2014). Innovation Projects Conducted by Distributed Teams: The Impact of Key Team Characteristics on Collaboration. Journal of Innovation Economics \& Management, 1, 37-72. 10.3917/ jie.013.0037

Díaz, C., Roncallo, L., López, M. y González, Z. (2018). Liderazgo: Consideraciones sobre su conceptualizació, evolución y retos ante la nueva realidad organizacional. Revista de Economía \& Adminsitración, 15(1), 1-18.

Escandon, D. y Hurtado, A. (2016). Influencia de los estilos de liderazgo en el desempeño de las empresas exportadoras colombianas. Revista Estudios Gerenciales, 32(139), 137-145.

Ganga, F. y Navarrete. (2013). Enfoques asociados al liderazgo eficaz para la organización. Revista Gaceta Laboral, 19(1), 52-77

Hernández-Sampieri, R. y Mendoza, C. (2018). Metodología de la investigación. Las rutas cuantitativa, cualitativa y mixta. Mc Graw Hill.

Huayta, C. L. y Luque, J. (2019), Inlfuencia del empowerment en el comrpomiso orgnizacional de los anlistas de crédito de las COOPAC en Arequipa [Tesis de pregrado, Universidad Católica de Santa María]. Repositorio de Tesis - Universidad Católica de Santa María. http://tesis.ucsm.edu.pe/repositorio/handle/ UCSM/9530

Lewin, K. (1939) Teoría del campo y experimentación en psicología social, Cuaderno del Instituto de Sociología de la Fac. de Filosofía de la UBA, (10)

López, F. y Chiclana, C. (2017). Engagement, una plataforma para el desarrollo de la persona. Revista Comunicación y Hombre, (14), 53-62.

Marcantonio, C. (2017). Concepto y alcance del término Engagement. Requerimiento a los empleados. Revista Argentina de Investigación en Negocios, 3(2), 81-91.
Martínez, M., Medin, C., Rodríguez, J., del Toro, E. y Rosa, I. (2016). El liderazgo como promotor del engagment en el trabajo. Alternativas cubanas en psicología, 4(10), $114-118$.

Pérez, B. I. y Valderrama, A. G. (2018). "estilos de liderazgo", felicidad y relación con el engagement en una organización del Gobierno Regional de Arequipa-Autodema-Majes Siguas [Tesis de pregrado, Universidad Nacional de San Agustín de Arequipa]. Repositorio Institucional Digital de la UNSA. http://repositorio. unsa.edu.pe/handle/UNSA/5857

Pérez, J. y Pedraza, X. (2018). Medición del work engagementy su relación con la comunicación, liderazgo y TIC en una empresa editorial mexicana. Revista SIGNOSInvestigación en sistemas de gestión, 11(1), 37-33.

Rodríguez, E., Pedraja, L. y Ganga, F. (2017). La relación entre los "estilos de liderazgo" y el desempeño de los equipos de dirección interna: un estudio exploratorio desde Chile. Revista Contabilidad y Negocios, 12(23), 1-18.

Sánchez, J. y Barraza, L. (2015). Percepciones sobre liderazgo. Revista Ra Ximhai, 11(4), 161-170.

Sanclemente, I., Elboj, C. y lñiquez T. (2017). Engagament o compromiso en profesionales de emfermería: variables socio-demográficas y laborales asociadas. Revista de Enfermería Global, 16(3), 400-413.

Vásquez, S., Benal, J. y Liesa, M. (2014). La conceptualización del liderazgo: una aproximación desde la práctica educativa. REICE. Revista Iberoamericana sobre Calidad, Eficiencia y Cambio en Educación., 12(5), 79-97.

Villalva, M. y Fierro, I. (2017). El liderazgo democrático: una aproximación conceptual. INNOVA. Research Journal, 2(4); 155-162

Zumaeta, F. (2018). Estilos de liderazgo y engagement en trabajadores de una empresa textil-retail del distrito de San Luis, 2018 [Tesis de pregrado, Universidad Cesar Vallejo]. Repositorio de la Universidad César Vallejo. https://hdl.handle.net/20.500.12692/30283

Contribución de los autores

AG-J: Todo el artículo

Fuentes de financiamiento.

La investigación fue realizada con recursos propios.

Conflictos de interés

No existe conflicto de interés.

\section{Correspondencia}

José Luis Arias Gonzáles

Dirección: Prolongación Avenida Ejercito 618, Cerro

Colorado, Arequipa, Perú.

CP.: 04000

Cel.: 967702156

Email: joseariasgon6@gmail.com 